\title{
Association between post-hospital clinic and telephone follow-up provider visits with 30-day readmission risk in an integrated health system
}

Huong Q. Nguyen ${ }^{1 *}$, Aileen Baecker ${ }^{1}$, Timothy Ho ${ }^{2}$, Dan N. Huynh ${ }^{2}$, Heather L. Watson ${ }^{2}$, Jing Li ${ }^{3}$ and Ernest Shen ${ }^{1}$

\begin{abstract}
Background: Follow-up visits with clinic providers after hospital discharge may not be feasible for some patients due to functional limitations, transportation challenges, need for physical distancing, or fear of exposure especially during the current COVID-19 pandemic.

Methods: The aim of the study was to determine the effects of post-hospital clinic (POSH) and telephone (TPOSH) follow-up provider visits versus no visit on 30-day readmission. We used a retrospective cohort design based on data from $1 / 1 / 2017$ to 12/31/2019 on adult patients $(n=213,513)$ discharged home from 15 Kaiser Permanente Southern California hospitals. Completion of POSH or TPOSH provider visits within 7 days of discharge was the exposure and all-cause 30-day inpatient and observation stay readmission was the primary outcome. We used matching weights to balance the groups and Fine-Gray subdistribution hazard model to assess for readmission risk.

Results: Unweighted all-cause 30-day readmission rate was highest for patients who completed a TPOSH (17.3\%) followed by no visit (14.2\%), non-POSH (evaluation and management visits that were not focused on the hospitalization: 13.6\%) and POSH (12.6\%) visits. The matching weighted models showed that the effects of POSH and TPOSH visits varied across patient subgroups. For high risk (LACE 11+) medicine patients, both POSH (HR: 0.77, 95\% Cl: $0.71,0.85, P<.001$ ) and TPOSH (HR: 0.91, 95\% Cl: 0.83, 0.99, $P=.03$ ) were associated with 23 and 9\% lower risk of 30-day readmission, respectively, compared to no visit. For medium to low risk medicine patients (LACE $<11)$ and all surgical patients regardless of LACE score or age, there were no significant associations for either visit type with risk of 30-day readmission.
\end{abstract}

Conclusions: Post-hospital telephone follow-up provider visits had only modest effects on 30-day readmission in high-risk medicine patients compared to clinic visits. It remains to be determined if greater use and comfort with virtual visits by providers and patients as a result of the pandemic might improve the effectiveness of these encounters.

Keywords: Care transition, Readmission, Post-discharge provider follow-up

\footnotetext{
* Correspondence: huong.q2.nguyen@kp.org

1 Department of Research and Evaluation, Kaiser Permanente Southern

California, Research and Evaluation, 100 S. Los Robles Avenue, 2nd Floor,

Pasadena, CA 91101, USA

Full list of author information is available at the end of the article
}

(c) The Author(s). 2021 Open Access This article is licensed under a Creative Commons Attribution 4.0 International License, which permits use, sharing, adaptation, distribution and reproduction in any medium or format, as long as you give appropriate credit to the original author(s) and the source, provide a link to the Creative Commons licence, and indicate if changes were made. The images or other third party material in this article are included in the article's Creative Commons licence, unless indicated otherwise in a credit line to the material. If material is not included in the article's Creative Commons licence and your intended use is not permitted by statutory regulation or exceeds the permitted use, you will need to obtain permission directly from the copyright holder. To view a copy of this licence, visit http://creativecommons.org/licenses/by/4.0/. The Creative Commons Public Domain Dedication waiver (http://creativecommons.org/publicdomain/zero/1.0/) applies to the data made available in this article, unless otherwise stated in a credit line to the data. 


\section{Introduction}

Transitional care management after hospital discharge typically includes a follow-up provider clinic visit within $7-14$ days depending on patient complexity $[1,2]$. We previously reported on the association between completion of a dedicated post-hospital (POSH) follow-up provider clinic visit with a $24 \%$ lower risk of 30-day inpatient readmissions in older patients within an integrated health system [3], a finding similar to other studies [4-6]. Nonetheless, since clinic visits were challenging for high risk patients who have functional limitations or no transportation, the health system began offering telephone POSH (TPOSH) appointments as an alternative to the clinic visits in 2015. Uptake of the TPOSH was limited and comprised only $7 \%$ of the postdischarge follow-up encounters prior to the COVID-19 pandemic.

In response to the dramatic shift to greater use of telehealth for routine clinic appointments out of necessity due to the COVID-19 pandemic [7], we aimed to advance the care transition evidence base by using data prior to the public health emergency to examine the effects of clinic (POSH) and telephone (TPOSH) visits completed within 7 days of discharge with a provider on 30-day inpatient and observation stay readmission compared to no visit and whether these effects varied by age, service line (medicine or surgical) and readmission risk as secondary analyses. In contrast to our earlier report [3], the analyses herein leverage a more contemporary cohort that reflects secular changes in transitional care management practices since 2014, is not restricted to older adults, and is not adversely affected by the disruptions in healthcare due to the COVID-19 pandemic.

\section{Methods}

\section{Study design and sample}

This retrospective cohort study included the first hospitalization for all adult patients who were discharged alive from 15 Kaiser Permanente Southern California (KPSC) hospitals between January 1, 2017 to December 31, 2019 to home or home health and remained enrolled in the health plan for at least 30 days post-discharge. This study was approved by the KPSC Institutional Review Board.

\section{Transitional care management}

Continuous performance improvement efforts across the 15 hospitals focused on ensuring that follow-up provider visits were scheduled within 7 days of discharge for medicine high risk patients as determined by a LACE score [8] of 11 or higher during this study period; hospitals with greater resources were encouraged to also schedule appointments for medium risk (LACE score 710) patients. Most POSH and TPOSH visits were scheduled before hospital discharge with all sites consistently achieving $>85 \%$ appointments scheduled for high risk patients. TPOSH visits may be offered by hospital scheduling staff to patients who they anticipated would have challenges with completing a clinic $\mathrm{POSH}$ visit or were offered by call center staff if patients reported that they could not attend their POSH clinic appointment. $\mathrm{POSH} / \mathrm{TPOSH}$ visits were flagged for the provider as being a post-hospital follow-up visit in the electronic medical record (EMR); reminders for routine care issues were suppressed during these visits. Providers were trained to focus on the post-discharge summary, medication review and issues that required follow-up. The $\mathrm{POSH}$ and TPOSH visits were generally $20 \mathrm{~min}$ in length. Other than the inability to conduct a physical assessment during a TPOSH, providers were expected to address standard transitional care needs of the patient. The TPOSH/POSH may take place before or after a standard 48-72-h post-discharge telephone call by an allied health staff. A non-POSH visit could have been scheduled before or after the hospitalization by the patient or a provider (primary care, specialist, and other clinicians) for any reason, including evaluation and management; the focus of these visits was not specifically on the hospitalization. Patients may have a follow-up visit with specialty care as a result of the hospitalization but due to challenges with access, these visits may not occur within the first 7 days of discharge.

\section{Covariates}

Socio-demographic (age, gender, race/ethnicity, being partnered), behavioral (no-show history in the last 12 months), social risk (receipt of medical financial assistance from the health plan in the year prior to admission), and clinical characteristics [risk for readmission or early death score based on length of stay, age, comorbidities, and emergency department visits in the prior 6 months (LACE category [8]), severity of the index hospitalization (LAPS2) [9], discharge disposition (home vs. home health), service line (medicine vs. surgical), functional status (non-ambulatory vs. ambulatory), falls risk (Schmid [10] score of 3+) within $24 \mathrm{~h}$ of discharge, and frailty category [11]] were obtained or calculated from the electronic medical record (EMR) system.

\section{Outcome}

The primary outcome was all-cause 30-day inpatient or observation stay readmission obtained from the EMR and claims.

\section{Statistical analysis}

Although the rate of death in the overall sample was low (1.5\%), the higher rate among the TPOSH group (3.6\%) indicated a need to account for death as a competing 
risk, using the subdistribution hazard approach of Fine and Gray [12]. We treated visit completion as a timedependent variable that could change in the first 7 days. While using time-varying covariates (i.e. visit completion) with the Fine-Gray subdistribution hazard model can preclude the estimation of their effect on either the subdistribution hazard or cumulative incidence function, visit completion as we defined it is fixed for all subsequent risk sets, and so is not subject to this limitation [13].

We used matching weights [14] where the propensity score (PS) were calculated using generalized boosted models $[15,16]$ to address confounding instead of regression adjustment, inverse probability of treatment weights (IPTW) or matching approaches. We chose to use matching weights for several reasons: 1 ) it provides the average treatment effect on the treated (ATT) interpretation of switching patients from one exposure group to another (unlike IPTW which estimates average treatment effects on the entire sample); 2) it mitigates the bias that results from using IPTW when some groups have extreme weights such as we observed for the TPOSH group, in which case the substantial nonoverlap on the PS [17] results in a violation of the common support assumption, by effectively assigning those with an extreme PS a weight close to 1; and 3) it avoids the nuances and complexity of matching on three or more groups to estimate the ATT, while also precluding the need to either exclude some patients from a matched analysis or introduce additional complexity by doing full matching $[14,18]$.

Covariates that were meaningfully associated with either visit completion or readmission [age, gender, race/ ethnicity, being partnered, no-show history in the last 12 months, receipt of medical financial assistance in the year prior to admission (a marker of social risk), risk for readmission or early death (LACE category [8]), severity of hospitalization (LAPS2) [9], discharge disposition (home vs. home health), service line (medicine vs. surgical), functional status (non-ambulatory vs. ambulatory) and fall risk (Schmid [10] score of 3+) within $24 \mathrm{~h}$ of discharge, frailty category [11], and hospital site] were included in the multinomial regression models to estimate propensity scores to construct the matching weights. Such associations were deemed meaningful using a combination of prior literature and examining statistical measures of association (namely the maximum standardized pairwise differences). Secondary subgroup analyses were stratified by age $(<65$ or $65+)$, service line (medicine vs. surgical) and LACE score $(<11$ : medium to low risk vs. 11+: high risk), to assess for heterogeneity of treatments effects. Analyses were performed with SAS Enterprise Guide 7.1 (Cary, North Carolina). A $P<.05$ was considered significant.

\section{Results}

\section{Patient flow}

The initial cohort of 390,365 adult, non-maternity patients discharged alive from 15 hospitals was pared down to a total of 213,513 unique patients who had their first index hospitalization during the study period after excluding 71,526 patients due to discharge disposition elsewhere not to home or home health, 9115 patients not enrolled for at least 30 days post discharge, and 96,211 patients with repeat hospitalizations during the study period (Fig. 1).

\section{Sample characteristics}

Approximately $30 \%$ of patients completed a POSH (26\%) or TPOSH (4\%) visit within 7-days of hospital discharge; $33 \%$ had a non-POSH visit and $37 \%$ did not have any clinic visit (Tables 1 and 2). Approximately 57\% of the high-risk medicine LACE patients for whom the hospitals prioritized for scheduling follow-up appointments completed a POSH or TPOSH visit within 7 days whereas $25 \%$ of the medium to low-risk medicine LACE patients completed such visits. Patients who completed a TPOSH tended to be older, had higher co-morbidities, frailty and readmission risk compared to patients completing other visit types or no visit. Surgical patients were more likely to complete nonPOSH visits or no visit compared to medicine patients. The maximum standardized differences in matching weights across all baseline variables were all $<0.10$ indicating acceptable balance across all six possible pairwise comparisons (POSH vs. no visit, TPOSH vs. no visit, nonPOSH vs. no visit, $\mathrm{POSH}$ vs. TPOSH, POSH vs. nonPOSH, TPOSH vs. nonPOSH) [19].

A total of $29,288(13.7 \%)$ and $3115(1.5 \%)$ of patients were readmitted or died within 30 days of being discharged alive, respectively. Among those patients who died within 30 days, 58\% had a readmission prior to death, while the remainder had a median survival time of 13 days (IQR: 6, 21). The unweighted all-cause 30-day readmission rate was highest for patients who completed a TPOSH (17.3\%) followed by no visit (14.2\%), nonPOSH (13.6\%) and POSH (12.6\%) (Table 2).

\section{Overall primary analyses}

For all patients, only POSH (HR: 0.83, 95\% CI: 0.77, $0.90, P<.001)$ provider visits completed within 7 days of hospital discharge were associated with a $17 \%$ lower risk of 30-day inpatient or observation readmission compared to no visit in the weighted models (Table 3). TPOSH provider visits were not associated with reductions in readmission risk for all patients (HR: 0.97, 95\% CI: $0.90,1.05, P=.48)$. POSH provider visits were associated with $15 \%$ lower risk of 30-day readmission 


\section{All adult, non-maternity discharges from 15 KPSC hospitals, Jan 1, 2017-Dec 31, 2019 $n=390,365$}

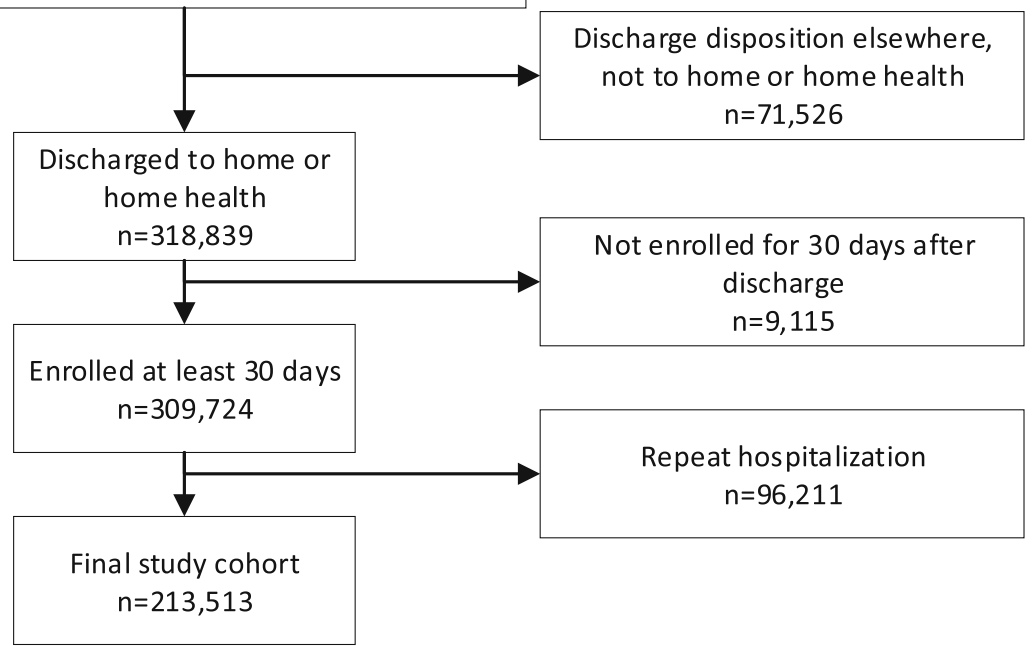

Fig. 1 Sample Flow

compared to TPOSH provider visits (HR: 0.85, 95\% CI: $0.79,0.93, P<.01)$.

\section{Secondary subgroup analyses}

For high risk (LACE 11+) medicine patients, both POSH (HR: 0.77, 95\% CI: 0.71, 0.85, $P<.001$ ) and TPOSH (HR: $0.91,95 \% \mathrm{CI}: 0.83,0.99, P=.03)$ provider visits were associated with 23 and 9\% lower risk of 30-day readmission, respectively, compared to no visit in the weighted models. For medium to low risk patients $(\mathrm{LACE}<11)$, neither POSH (HR: 0.84, 95\% CI: 0.67, 1.05, $P=.12$ ) nor TPOSH (HR: 1.14, 95\%CI: 0.94, 1.39, $P=0.19$ ) provider visits were associated with lower readmission risk compared to no visit.

For all surgical patients regardless of LACE score or age, there were no significant associations for any visit type with risk of 30-day readmission in the weighted models.

\section{Discussion}

Using a more contemporary and diverse cohort of patients discharged from hospital to home or home health, we found that completion of either a clinic or telephone provider follow-up visit within 7 days of discharge was associated with lower 30-day readmission compared to no visit only for high risk medicine patients. The risk reduction for $\mathrm{POSH}$ provider visits was comparable to our previous report [3] while the TPOSH effects though statistically significant, were marginal at best. A surprising finding was that none of the visit types were associated with lower readmission for lower risk medicine and all surgical patients.

Although the TPOSH was introduced to address a gap for patients who have mobility and transportation challenges and who would otherwise not have any follow-up provider visit, the limited effects of TPOSH compared to POSH were not surprising for several reasons. Clinic providers may have had insufficient practice with telephonic transitional care management prior to the pandemic as demonstrated in the small volume of TPOSH visits and may have been more cautious in directing patients to seek hospital-based care for worsening symptoms. Other ancillary team members were not able to augment the provider's care as they would during a clinic visit. Neither physical exams nor visual assessments were performed during these encounters and thus providers were limited to patient and/or family selfreport. With as many as half of elderly hospitalized patients having cognitive impairment [20], and possibly, without the assistance of a capable family member, gaps in communication and early detection of decompensation may be problematic. Use of video and/or remote assessments or biometrics could potentially enhance the effectiveness of these telephone encounters, especially for more complex, higher-risk older patients but only if family can assist with these technologies [21, 22].

The COVID-19 pandemic has accelerated adoption of digital technologies [7, 23] across all aspects of health care, especially with physically-distanced care and the earlier discharge of COVID-19 patients to increase hospital capacity [24]. Learnings from use of digital home- 
Table 1 Baseline sample characteristics by completion of post-hospital follow-up provider visits within 7-days of discharge

\begin{tabular}{|c|c|c|c|c|c|c|c|}
\hline & POSH & TPOSH & Non-POSH & No Visit & Total & $\operatorname{Max} \mathrm{S}$ & Diff \\
\hline & $\begin{array}{l}n=56,383 \\
(26.4 \%)\end{array}$ & $\begin{array}{l}n=9009 \\
(4.2 \%)\end{array}$ & $\begin{array}{l}n=69,997 \\
(32.8 \%)\end{array}$ & $\begin{array}{l}n=78,124 \\
(36.6 \%)\end{array}$ & $n=213,513$ & $\begin{array}{l}\text { Not } \\
\text { Wgtd }\end{array}$ & Wgtd \\
\hline Socio-demographics & & & & & & & \\
\hline Age & $66.5(16.4)$ & $69.5(16.2)$ & $58.9(17.8)$ & $57.8(18.6)$ & $60.9(18.1)$ & 0.68 & 0.05 \\
\hline Female & $27,203(48.2 \%)$ & 4857 (53.9\%) & $36,903(52.7 \%)$ & 45,197 (57.9\%) & $114,160(53.5 \%)$ & 0.10 & 0.01 \\
\hline Race/Ethnicity & & & & & & & \\
\hline Asian & $5042(8.9 \%)$ & $721(8 \%)$ & $6432(9.2 \%)$ & $6459(8.3 \%)$ & $18,654(8.7 \%)$ & 0.01 & 0.00 \\
\hline Black & $6440(11.4 \%)$ & $1446(16.1 \%)$ & $7434(10.6 \%)$ & $9146(11.7 \%)$ & $24,466(11.5 \%)$ & 0.05 & 0.01 \\
\hline Hispanic & $18,788(33.3 \%)$ & $2517(27.9 \%)$ & $24,815(35.5 \%)$ & $28,760(36.8 \%)$ & $74,880(35.1 \%)$ & 0.09 & 0.01 \\
\hline Other & $1041(1.8 \%)$ & $155(1.7 \%)$ & $1385(2 \%)$ & $1554(2 \%)$ & 4135 (1.9\%) & 0.00 & 0.00 \\
\hline White & $25,072(44.5 \%)$ & $4170(46.3 \%)$ & $29,931(42.8 \%)$ & 32,205 (41.2\%) & $91,378(42.8 \%)$ & 0.05 & 0.01 \\
\hline Marital status: Partnered & $32,842(58.2 \%)$ & $4622(51.3 \%)$ & $42,151(60.2 \%)$ & $44,166(56.5 \%)$ & $123,781(58 \%)$ & 0.09 & 0.01 \\
\hline Education: < College & $41,216(73.1 \%)$ & $6504(72.2 \%)$ & $50,048(71.5 \%)$ & $56,640(72.5 \%)$ & $154,370(72.3 \%)$ & 0.09 & 0.05 \\
\hline Household income: $>\$ 20,000$ & $48,602(86.2 \%)$ & $7667(85.1 \%)$ & $60,477(86.4 \%)$ & $67,187(86.0 \%)$ & $184,048(86.2 \%)$ & 0.15 & 0.06 \\
\hline Spoken language: English & $48,993(86.9 \%)$ & $8108(90 \%)$ & $62,222(88.9 \%)$ & $69,453(88.9 \%)$ & $188,776(88.4 \%)$ & 0.03 & 0.02 \\
\hline Insurance type ${ }^{a}$ & & & & & & & \\
\hline Commercial/private pay & $21,503(38.1 \%)$ & $2772(30.8 \%)$ & $37,651(53.8 \%)$ & $43,070(55.1 \%)$ & $104,996(49.2 \%)$ & 0.24 & 0.02 \\
\hline Dual & $3215(5.7 \%)$ & $591(6.6 \%)$ & $2450(3.5 \%)$ & $2907(3.7 \%)$ & $9163(4.3 \%)$ & 0.03 & 0.01 \\
\hline Medicaid & $2493(4.4 \%)$ & $392(4.4 \%)$ & $3838(5.5 \%)$ & $4754(6.1 \%)$ & $11,477(5.4 \%)$ & 0.02 & 0.01 \\
\hline Medicare & $29,160(51.7 \%)$ & $5250(58.3 \%)$ & $26,035(37.2 \%)$ & $27,330(35 \%)$ & $87,775(41.1 \%)$ & 0.23 & 0.02 \\
\hline Received medical financial assistance in prior year & $4590(8.1 \%)$ & $941(10.4 \%)$ & $5163(7.4 \%)$ & $5035(6.4 \%)$ & $15,729(7.4 \%)$ & 0.04 & 0.00 \\
\hline Health and Behavior Before Hospitalization & & & & & & & \\
\hline Clinic no show rate (at least once) & $34,084(60.5 \%)$ & $6185(68.7 \%)$ & $43,594(62.3 \%)$ & $47,297(60.5 \%)$ & $131,160(61.4 \%)$ & 0.08 & 0.01 \\
\hline Charlson comorbidity index ${ }^{\mathrm{b}}$ & $4.7(3.08)$ & $5.5(3.14)$ & $3.8(2.99)$ & $3.4(2.94)$ & $4.0(3.07)$ & 0.68 & 0.03 \\
\hline Myocardial infarction & $9635(17.1 \%)$ & $1727(19.2 \%)$ & $7030(10 \%)$ & $6703(8.6 \%)$ & $25,095(11.8 \%)$ & - & - \\
\hline Congestive heart failure & $13,396(23.8 \%)$ & $2684(29.8 \%)$ & $9295(13.3 \%)$ & $8613(11 \%)$ & $33,988(15.9 \%)$ & - & - \\
\hline Peripheral vascular disease & $26,973(47.8 \%)$ & $5236(58.1 \%)$ & $22,050(31.5 \%)$ & $22,215(28.4 \%)$ & $76,474(35.8 \%)$ & - & - \\
\hline Cerebrovascular disease & $12,175(21.6 \%)$ & $2358(26.2 \%)$ & $9105(13 \%)$ & $9833(12.6 \%)$ & $33,471(15.7 \%)$ & - & - \\
\hline Dementia & $3536(6.3 \%)$ & $1134(12.6 \%)$ & $1940(2.8 \%)$ & $3357(4.3 \%)$ & $9967(4.7 \%)$ & - & - \\
\hline Rheumatic disease & $3937(7 \%)$ & $710(7.9 \%)$ & $3692(5.3 \%)$ & $3711(4.8 \%)$ & $12,050(5.6 \%)$ & - & - \\
\hline Peptic ulcer disease & $3779(6.7 \%)$ & $687(7.6 \%)$ & $3094(4.4 \%)$ & $3296(4.2 \%)$ & $10,856(5.1 \%)$ & - & - \\
\hline Mild liver disease & $8727(15.5 \%)$ & $1499(16.6 \%)$ & $9095(13 \%)$ & $9301(11.9 \%)$ & $28,622(13.4 \%)$ & - & - \\
\hline Moderate or severe liver disease & $1355(2.4 \%)$ & $236(2.6 \%)$ & $890(1.3 \%)$ & $837(1.1 \%)$ & $3318(1.6 \%)$ & - & - \\
\hline Diabetes without chronic complication & $7821(13.9 \%)$ & $1159(12.9 \%)$ & $8766(12.5 \%)$ & $9464(12.1 \%)$ & $27,210(12.7 \%)$ & - & - \\
\hline Diabetes with chronic complication & $18,284(32.4 \%)$ & $3201(35.5 \%)$ & $14,142(20.2 \%)$ & $12,878(16.5 \%)$ & $48,505(22.7 \%)$ & - & - \\
\hline Hemiplegia or paraplegia & $2909(5.2 \%)$ & $740(8.2 \%)$ & $2211(3.2 \%)$ & $2721(3.5 \%)$ & $8581(4 \%)$ & - & - \\
\hline Renal disease & $20,089(35.6 \%)$ & $3776(41.9 \%)$ & $14,696(21 \%)$ & $14,609(18.7 \%)$ & $53,170(24.9 \%)$ & - & - \\
\hline Any malignancy (lymphomas, leukemias etc) & $8825(15.7 \%)$ & $1788(19.8 \%)$ & $11,963(17.1 \%)$ & $9563(12.2 \%)$ & $32,139(15.1 \%)$ & - & - \\
\hline Metastatic solid tumor & $3343(5.9 \%)$ & $830(9.2 \%)$ & $4578(6.5 \%)$ & $3006(3.8 \%)$ & $11,757(5.5 \%)$ & - & - \\
\hline AIDS/HIV & $236(0.4 \%)$ & $51(0.6 \%)$ & $281(0.4 \%)$ & $244(0.3 \%)$ & $812(0.4 \%)$ & - & - \\
\hline Frailty index & $8.8(6.65)$ & $11.1(8.35)$ & $6.1(6.05)$ & $5.7(6.44)$ & $6.9(6.65)$ & & \\
\hline Low risk $<5$ & $18,052(32 \%)$ & $2310(25.6 \%)$ & $37,893(54.1 \%)$ & $46,855(60 \%)$ & $105,110(49.2 \%)$ & 0.34 & 0.01 \\
\hline Intermediate risk 5-14 & $29,643(52.6 \%)$ & $4366(48.5 \%)$ & $26,225(37.5 \%)$ & $24,610(31.5 \%)$ & $84,844(39.7 \%)$ & 0.21 & 0.01 \\
\hline High Risk 15+ & $8688(15.4 \%)$ & $2333(25.9 \%)$ & $5879(8.4 \%)$ & $6659(8.5 \%)$ & $23,559(11 \%)$ & 0.17 & 0.01 \\
\hline
\end{tabular}

Values are presented as either mean (SD) or $n$ (column \%), unless otherwise indicated

POSH Post-hospital follow-up clinic visit, TPOSH Post-hospital follow-up telephone visit, nonPOSH visit not related to post-hospital follow-up

Wgtd Weighted maximum standardized differences across groups

a Missingness $<0.1$

${ }^{b}$ Weighting only done on index, not individual conditions 
Table 2 Characteristics of the index hospitalization, 30-day readmission and mortality

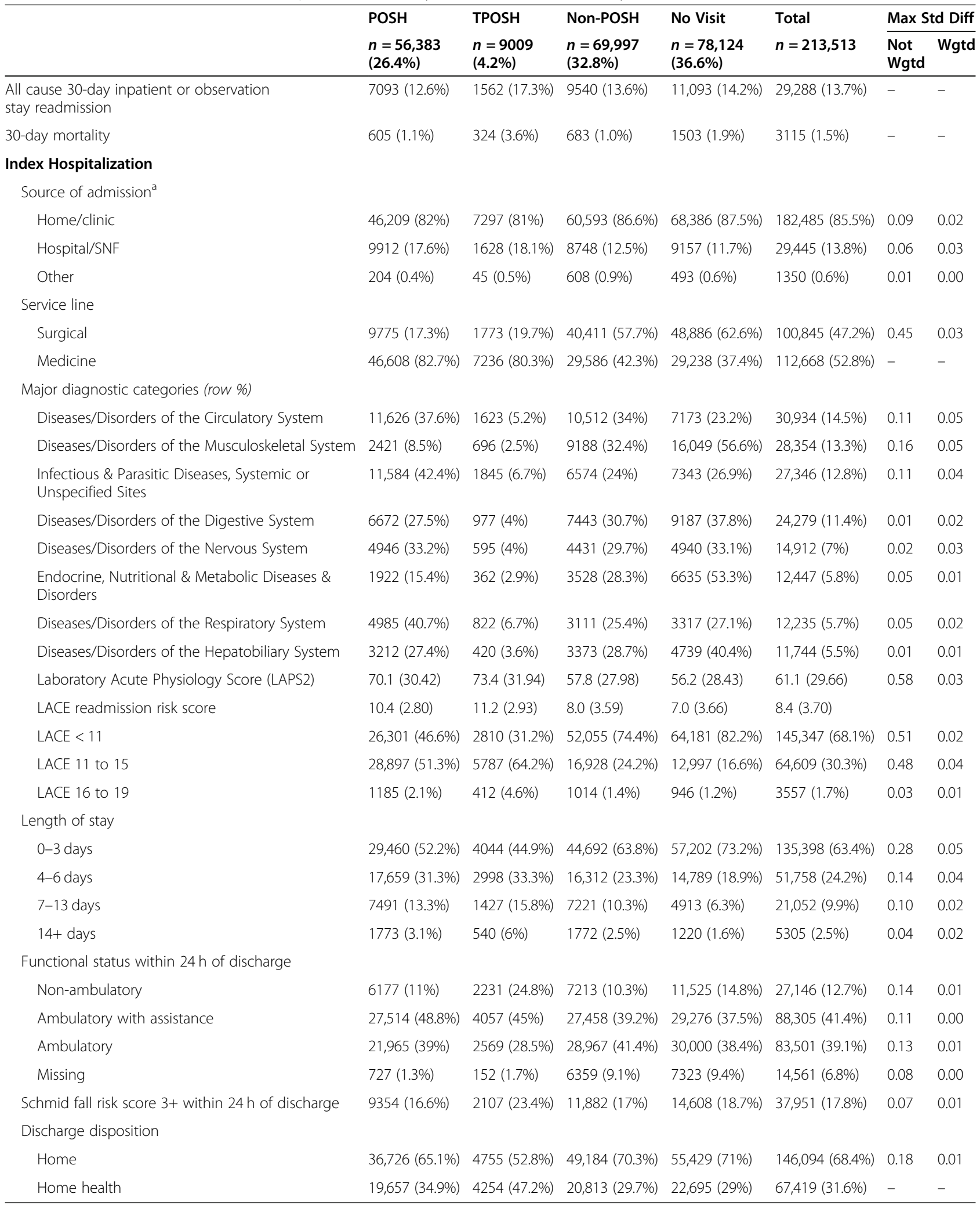

Values are presented as either mean (SD) or $n$ (column \%), unless otherwise indicated

POSH Post-hospital follow-up clinic visit, TPOSH Post-hospital follow-up telephone visit, nonPOSH visit not related to post-hospital follow-up

Wgtd Weighted maximum standardized differences across groups

a Missingness $<0.1$ 
Table 3 Weighted models examining the effect of post-hospital follow-up provider visits completed within 7-days of discharge on all cause 30-day inpatient and observation stay readmission stratified by age, service line, and readmission risk

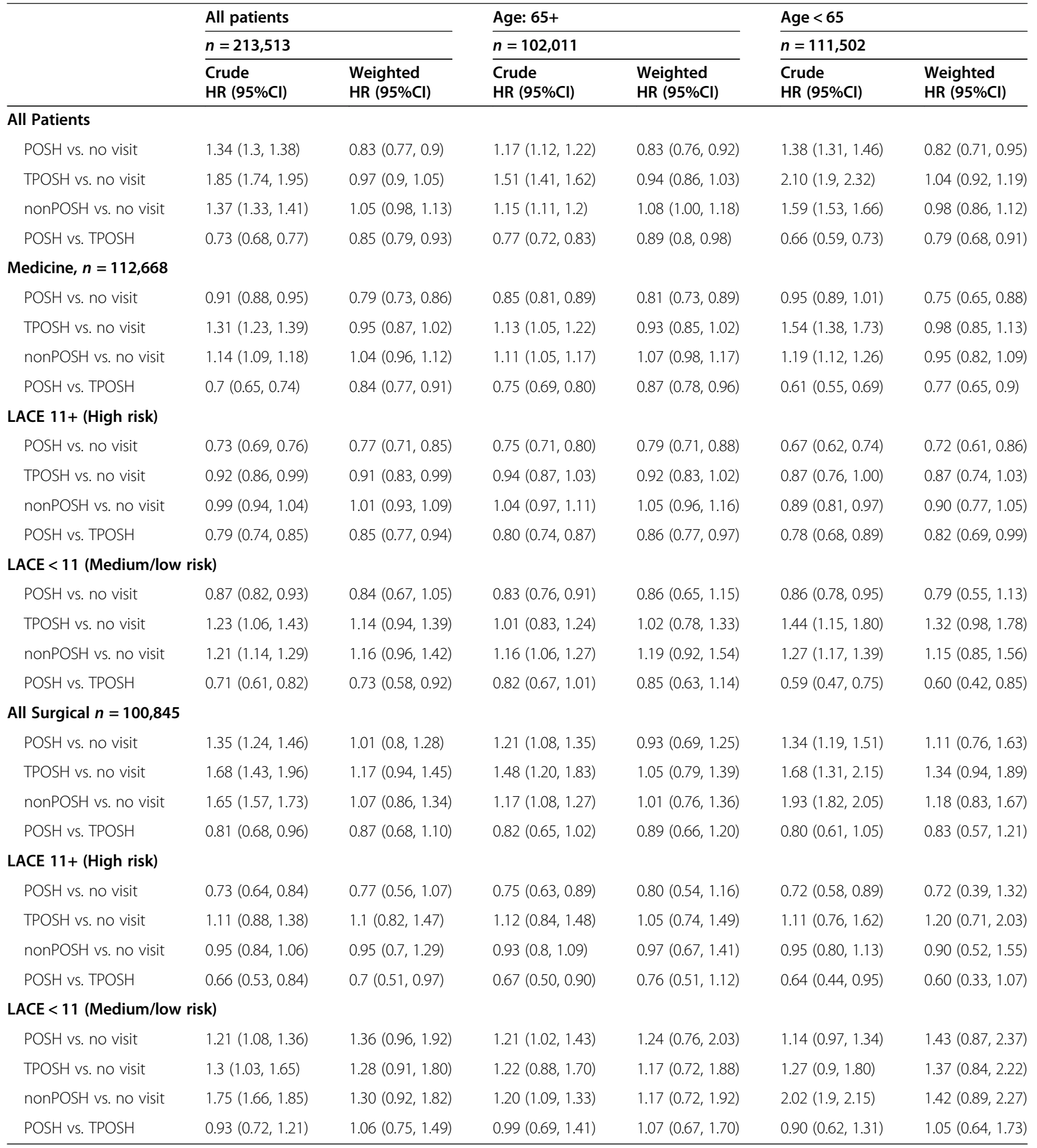

Values are presented as hazard ratio (HR) and $95 \%$ confidence intervals

POSH Post-hospital follow-up clinic visit, TPOSH Telephone post-hospital follow-up visit, nonPOSH Visit not related to post-hospital follow-up, LACE Length of stay; Acuity of admission; Co-morbidities; Emergency visits in previous 6 months

Matching weights were used, based on multinomial propensity score models that included age, gender, race/ethnicity, being partnered, no-show history in the last 12 months, receipt of medical financial assistance in the year prior to admission (a marker of social risk), frailty category, risk for readmission or early death (LACE category), severity of hospitalization (LAPS2), service line (medicine vs. surgical), functional status (non-ambulatory, ambulatory with assistance, or ambulatory) and fall risk (Schmid score of $3+$ ) within $24 \mathrm{~h}$ of discharge; disposition (home vs. home health), and hospital site 
monitoring technologies for transitional care with COVID-19 patients could serve as exemplars for optimizing transitional care approaches for other medical conditions in the future. Since the start of the pandemic through December 2020, this health system conducted $87 \%$ of the transitional care management visits via telephone, $3 \%$ by video, and $10 \%$ in person, a complete flip compared to the pre-pandemic era. While there are no other published reports specifically on virtual care transition management practices, Eberly et al. [25] reported a more balanced distribution of video (45\%) and telephone (55\%) primary and specialty care visits from a large academic health system from March to May 2020. We expect that video visits will increase over time with greater provider comfort and increased patient access to technology [26] and also due to telehealth reimbursement for Medicare Advantage plans which took effect in January 1, 2020 but the long-term future of telehealth reimbursement in fee-for-service Medicare remains to be seen after the end of the public health emergency [23].

The finding that completion of any follow-up visit whether with a provider or ancillary care staff within 7 days of hospital discharge was not associated with lower readmission risk compared to no visit for surgical patients merit more detailed analyses by types of surgical procedures (high vs. low risk, planned vs. unplanned) to determine the optimal timing and appropriate type of provider follow-up post hospitalization in order to maximize value for health systems and payers [27]. In contrast to medicine patients, nearly half of the surgical patients did not have an outpatient follow-up visit within a week of discharge. It is possible that because the vast majority (87\%) of these patients were considered as lower risk for readmission (LACE scores $<11$ ), follow-up visits were rightly not prioritized by the discharging providers as our analyses showed that these visits were not associated with any added benefit in terms of risk reduction.

Strengths of this study included use of a large, diverse contemporary cohort that was exposed to many changes in the care transition management practices in recent years, replication of findings from our previous, limited analyses of an older study cohort, ability to balance relevant baseline covariates across comparison groups using matching weights, and inclusion of both inpatient and observation stay readmissions as a primary outcome in anticipation of changes to how readmissions are counted in the Center for Medicare and Medicaid Services Hospital Readmission Reduction Program as of January 1, 2021.

\section{Limitations}

Since this analysis was limited to patients discharged from Kaiser Permanente hospitals to home, the findings may not generalize to patients discharged to other higher-level settings or in non-integrated care systems where electronic records are not easily shared across hospital and ambulatory care settings. The TPOSH volume was relatively small for several of the subgroups, especially for younger patients and those on the surgical service, to obtain a reliable estimate of benefit or harm. Since the care transition quality improvement efforts in this health system did not distinguish across disease states nor cause of the index admission except for medicine vs. surgical, we did not explore additional subgroup analyses in this paper though this should be the focus of future study. The matching weights were balanced on measured covariates but the cohorts may differ in other ways; omission of unmeasured confounders such as exposure to other care transition interventions and treatment adherence, as well selection bias, are other notable limitations of this observational study design. Nonetheless, prior studies of heart failure and COPD for instance, have also reported an association between completion of follow-up provider visits within 7 days post-discharge and lower rates of 30-day readmission [28, 29]. We did not account for the multiple comparisons in the subgroup analyses and thus some of the statistically significant findings may be spurious. However, we were cautious with our interpretation of these results. Finally, we were not able to assess the frequency of clinical escalations associated with the TPOSH visits.

\section{Conclusions}

We found that completion of either a clinic or telephone provider follow-up visit within 7 days of hospital discharge was associated with lower 30-day inpatient or observation readmission compared to no visit only for high risk medicine patients. The effects of the telephone provider follow-up, though statistically significant, were marginal at best during the pre-pandemic era. It remains to be determined if greater use and comfort with virtual visits by providers and patients as a result of the pandemic might improve the effectiveness of these encounters. The dramatic transformation of care delivery to virtual care in response to the COVID-19 pandemic offers an unprecedented opportunity to further examine the right mix of in-person clinic or home-based and remote care (telephone, video, and e-visits) for posthospital discharge follow-up across multiple relevant patient subgroups [30] to ensure equitable access to high quality care transition services $[25,31,32]$.

\section{Acknowledgements}

We thank Ms. Janet Lee for her assistance in acquiring the data and Dr. Harun Kundi from the Department of Medicine, Beth Israel Deaconess Medical Center, Boston, Massachusetts for sharing the frailty index code.

\section{Authors' contributions}

Drs. Baecker, Shen and Nguyen had full access to all of the data in the study and takes responsibility for the integrity of the data and the accuracy of the 
data analysis. Study concept and design: Nguyen, Baecker, Ho, Huynh, Watson. Acquisition, analysis, or interpretation of data: Nguyen, Baecker, Ho, Huynh, Watson, Li, Shen. Drafting of the manuscript: Nguyen, Baecker. Critical revision of the manuscript for important intellectual content: Nguyen, Baecker, Ho, Huynh, Watson, Li, Shen. Statistical analysis: Baecker and Shen. Study supervision: Nguyen. The author(s) read and approved the final manuscript.

\section{Funding}

Kaiser Permanente Southern California to the Care Improvement Research Team (CIRT)

\section{Availability of data and materials}

The datasets generated and/or analysed during the current study are not publicly available due to the identifiable nature of the data but may be available in anonymized form from the corresponding author under the following conditions: (1) agreement to collaborate with the study team on all publications, (2) provision of external funding to anonymize the data and for administrative and investigator time necessary for this collaboration, (3) demonstration that the external investigative team is qualified and has documented evidence of training for human subjects protections, and (4) agreement to abide by the terms outlined in data use agreements between institutions.

\section{Declarations}

\section{Ethics approval and consent to participate}

This study was approved by the Kaiser Permanente Southern California Institutional Review Board (\#10594). Patient written informed consent was waived by the KPSC IRB. All methods were carried out in accordance with relevant guidelines and regulations.

\section{Consent for publication}

Not applicable.

\section{Competing interests}

The authors have declared no conflicts of interest in relation to this paper.

\section{Author details}

'Department of Research and Evaluation, Kaiser Permanente Southern California, Research and Evaluation, 100 S. Los Robles Avenue, 2nd Floor, Pasadena, CA 91101, USA. 'Kaiser Permanente Southern California, Regional Clinical Operations, Pasadena, USA. ${ }^{3}$ University of Kentucky, Lexington, USA.

Received: 10 May 2021 Accepted: 28 July 2021

Published online: 17 August 2021

\section{References}

1. Hansen LO, Young RS, Hinami K, Leung A, Williams MV. Interventions to reduce 30-day rehospitalization: a systematic review. Ann Intern Med. 2011; 155(8):520-8. https://doi.org/10.7326/0003-4819-155-8-201110180-00008.

2. Leppin AL, Gionfriddo MR, Kessler M, Brito JP, Mair FS, Gallacher K, et al. Preventing 30-day hospital readmissions: a systematic review and Metaanalysis of randomized trials. JAMA Intern Med. 2014;174(7):1095-107. https://doi.org/10.1001/jamainternmed.2014.1608.

3. Shen E, Koyama SY, Huynh DN, Watson HL, Mittman B, Kanter MH, et al. Association of a Dedicated Post-Hospital Discharge Follow-up Visit and 30day readmission risk in a Medicare advantage population. JAMA Intern Med. 2017:177(1):132-5. https://doi.org/10.1001/jamainternmed.2016.7061.

4. Hernandez AF, Greiner MA, Fonarow GC, Hammill BG, Heidenreich PA, Yancy CW, et al. Relationship between early physician follow-up and 30-day readmission among Medicare beneficiaries hospitalized for heart failure. JAMA. 2010;303(17):1716-22. https://doi.org/10.1001/jama.2010.533.

5. Lee KK, Yang J, Hernandez AF, Steimle AE, Go AS. Post-discharge follow-up characteristics associated with 30-day readmission after heart failure hospitalization. Med Care. 2016;54(4):365-72. https://doi.org/10.1097/MLR. 0000000000000492.

6. Tung YC, Chang GM, Chang HY, Yu TH. Relationship between early physician follow-up and 30-day readmission after acute myocardial infarction and heart failure. PLoS One. 2017;12(1):e0170061. https://doi.org/1 0.1371/journal.pone.0170061.
7. Hollander JE, Carr BG. Virtually perfect? Telemedicine for Covid-19. N Engl J Med. 2020;382(18):1679-81. https://doi.org/10.1056/NEJMp2003539.

8. van Walraven C, Dhalla IA, Bell C, Etchells E, Stiell IG, Zarnke K, et al. Derivation and validation of an index to predict early death or unplanned readmission after discharge from hospital to the community. CMAJ. 2010; 182(6):551-7. https://doi.org/10.1503/cmaj.091117.

9. Escobar GJ, Gardner MN, Greene JD, Draper D, Kipnis P. Risk-adjusting hospital mortality using a comprehensive electronic record in an integrated health care delivery system. Med Care. 2013;51(5):446-53. https://doi.org/1 0.1097/MLR.0b013e3182881c8e

10. Schmid NA. Reducing patient falls: a research-based comprehensive fall prevention program. Mil Med. 1990;155(5):202-7. https://doi.org/10.1093/ milmed/155.5.202.

11. Kundi H, Wadhera RK, Strom JB, Valsdottir LR, Shen C, Kazi DS, et al. Association of Frailty with 30-day outcomes for acute myocardial infarction, heart failure, and pneumonia among elderly adults. JAMA Cardiol. 2019; 4(11):1084-91. https://doi.org/10.1001/jamacardio.2019.3511.

12. Fine JP, Gray RJ. A proportional hazards model for the subdistribution of a competing risk. J Am Stat Assoc. 1999;94(446):496-509. https://doi.org/10.1 080/01621459.1999.10474144.

13. Austin PC, Latouche A, Fine JP. A review of the use of time-varying covariates in the Fine-Gray subdistribution hazard competing risk regression model. Stat Med. 2020;39(2):103-13. https://doi.org/10.1002/sim.8399.

14. Yoshida K, Hernandez-Diaz S, Solomon DH, et al. Matching weights to simultaneously compare three treatment groups: comparison to three-way matching. Epidemiology. 2017;28(3):387-95. https://doi.org/10.1097/EDE. 0000000000000627

15. McCaffrey DF, Griffin BA, Almirall D, Slaughter ME, Ramchand R, Burgette LF. A tutorial on propensity score estimation for multiple treatments using generalized boosted models. Stat Med. 2013;32(19):3388-414. https://doi. org/10.1002/sim.5753.

16. Toolkit for weighting and analysis of nonequivalent groups: A tutorial for the TWANG SAS Macros [computer program]. 2016.

17. Franklin JM, Eddings W, Austin PC, Stuart EA, Schneeweiss S. Comparing the performance of propensity score methods in healthcare database studies with rare outcomes. Stat Med. 2017;36(12):1946-63. https://doi.org/10.1002/ $\operatorname{sim} .7250$.

18. Austin PC, Stuart EA. The performance of inverse probability of treatment weighting and full matching on the propensity score in the presence of model misspecification when estimating the effect of treatment on survival outcomes. Stat Methods Med Res. 2015;26(4):1654-70.

19. Austin PC. Balance diagnostics for comparing the distribution of baseline covariates between treatment groups in propensity-score matched samples. Stat Med. 2009;28(25):3083-107. https://doi.org/10.1002/sim.3697.

20. Mukadam N, Sampson EL. A systematic review of the prevalence, associations and outcomes of dementia in older general hospital inpatients. Int Psychogeriatr. 2011;23(3):344-55. https://doi.org/10.1017/S104161021 0001717.

21. Dewar $\mathrm{S}$, Lee $P G$, Suh $\Pi$, Min L. Uptake of virtual visits in a geriatric primary care clinic during the COVID-19 pandemic. J Am Geriatr Soc. 2020;68(7): 1392-4. https://doi.org/10.1111/jgs.16534.

22. Nouri S, Khoong EC, Lyles CR, Karliner L. Addressing Equity in Telemedicine for Chronic Disease Management During the Covid-19 Pandemic. NEJM Catalyst. 2020; https://catalyst.nejm.org/doi/full/10.1056/CAT.20.0123.

23. Perakslis E, Ginsburg GS. Digital Health-the need to assess benefits, risks, and value. JAMA. 2020. https://doi.org/10.1001/jama.2020.22919.

24. Morgan A, Balachandran M, Do D, et al. Remote monitoring of patients with Covid-19: design, implementation, and outcomes of the first 3,000 patients in COVID watch. NEJM Catalyst. 2020; https://catalyst.nejm.org/doi/full/10.1 056/CAT20.0342

25. Eberly LA, Kallan MJ, Julien HM, Haynes N, Khatana SAM, Nathan AS, et al. Patient characteristics associated with telemedicine access for primary and specialty ambulatory care during the COVID-19 pandemic. JAMA Netw Open. 2020;3(12):e2031640. https://doi.org/10.1001/jamanetworkopen.2020.31640.

26. Zulman DM, Verghese A. Virtual care, telemedicine visits, and real connection in the era of COVID-19: unforeseen opportunity in the face of adversity. JAMA. 2021;325(5):437-8. https://doi.org/10.1001/jama.2020.27304.

27. Brooke BS, Stone DH, Cronenwett JL, Nolan B, DeMartino RR, MacKenzie TA et al. Early primary care provider follow-up and readmission after high-risk surgery. JAMA Surg. 2014;149(8):821-8. https://doi.org/10.1001/jamasurg.2 014.157. 
28. Health Quality O. Effect of early follow-up after hospital discharge on outcomes in patients with heart failure or chronic obstructive pulmonary disease: a systematic review. Ont Health Technol Assess Ser. 2017;17(8):1-37.

29. Edmonston DL, Wu J, Matsouaka RA, Yancy C, Heidenreich P, Piña IL, et al. Association of post-discharge specialty outpatient visits with readmissions and mortality in high-risk heart failure patients. Am Heart J. 2019;212:10112. https://doi.org/10.1016/j.ahj.2019.03.005.

30. Hollander JE, Sites FD. The transition from reimagining to recreating Health care is now. 2020; https://catalyst.nejm.org/doi/full/10.1056/CAT.20.0093. Accessed 26 Apr 2020.

31. Lam K, Lu AD, Shi Y, Covinsky KE. Assessing Telemedicine Unreadiness Among Older Adults in the United States During the COVID-19 Pandemic. JAMA Intern Med. 2020; Published online August 3, 2020. https://doi.org/1 0.1001/jamainternmed.2020.2671.

32. Roberts ET, Mehrotra A. Assessment of Disparities in Digital Access Among Medicare Beneficiaries and Implications for Telemedicine. JAMA Intern Med. 2020; Published online August 3, 2020. https://doi.org/10.1001/jama internmed.2020.2666.

\section{Publisher's Note}

Springer Nature remains neutral with regard to jurisdictional claims in published maps and institutional affiliations.

- fast, convenient online submission

- thorough peer review by experienced researchers in your field

- rapid publication on acceptance

- support for research data, including large and complex data types

- gold Open Access which fosters wider collaboration and increased citations

- maximum visibility for your research: over $100 \mathrm{M}$ website views per year

At $\mathrm{BMC}$, research is always in progress.

Learn more biomedcentral.com/submissions 